\title{
Probing the Skin of a Lead Nucleus
}

\author{
Researchers make the most precise measurement yet of the neutron \\ distribution in a heavy nucleus, with implications for the structure of \\ neutron stars.
}

By Kate Scholberg

$\square$ opular cartoon visualizations depict the protons and neutrons in a nucleus as colored marbles packed randomly into a sphere. In reality, heavy nuclei-in which neutrons tend to outnumber protons-are more differentiated, with the neutrons nudged radially outward. At the outer limits of such nuclei, the neutrons form a thin "skin" enclosing a core of mixed neutrons and protons (Fig. 1). Now, the Lead Radius Experiment (PREX) Collaboration at the Thomas Jefferson National Accelerator Facility in Virginia has determined the thickness of this neutron-rich skin in lead-208, a stable isotope with 44 more neutrons than protons [1]. The measurement, which addresses questions relating to all four fundamental forces of nature, yields insight into the structure of neutron stars, and it will have far-reaching implications for multimessenger astronomy and particle physics.
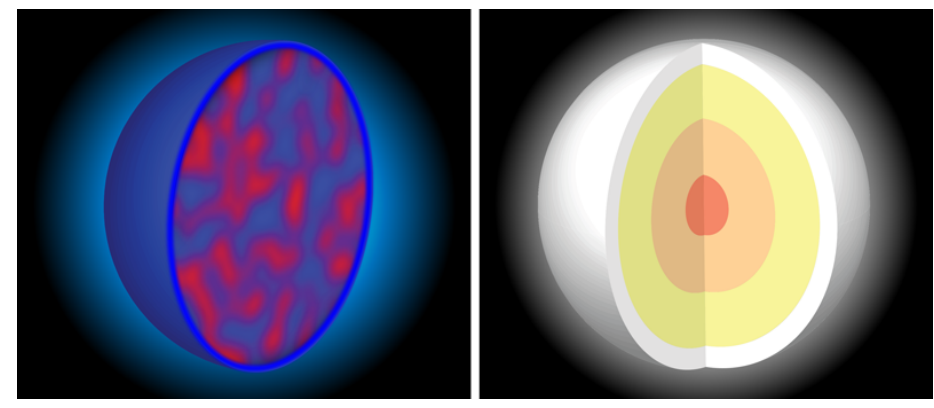

Figure 1: A cartoon image of a lead-208 nucleus, showing the mixed proton-neutron core and the neutron "skin" (left). Measuring the thickness of the neutron skin offers clues about how neutron stars are structured (right).

Credit: APS/Alan Stonebraker
The spatial distribution of protons within nuclei is well understood as a result of decades of scattering experiments with electromagnetic probes. Obtaining a similar understanding for neutrons is more challenging, as these neutral particles are mostly invisible to electrically charged probes. Given that neutrons do interact via the strong force, their nuclear distributions are, in principle, discoverable using strongly charged hadronic probes. However, strong interactions, described by quantum chromodynamics, have large theoretical uncertainties, and precise measurements using such methods are therefore difficult to achieve in practice.

Another approach is to leverage weak scattering, and techniques based on this interaction are much more effective. The reason for that is twofold: Weak scattering is far better understood than the scattering caused by strong interactions, and the nucleus's weak charge-in contrast to its electromagnetic one-is dominated by its neutron content. But the weak interaction is much weaker than the electromagnetic interaction, which means that its subtle effects on electron-nucleus scattering must be teased out carefully.

These subtle effects arise from a special feature of the weak interaction that gives it its signature parity violation: the strength of the interaction depends on a spatial direction. When electrons scatter from neutrons in the nucleus, they exchange $Z$ bosons, the weak-force carrier particles. When the electrons are polarized, the scattering process is asymmetric, with left-handed electrons (whose spins are in antialignment with their momentum) scattering off nuclei slightly less often than right-handed ones. The size of the asymmetry is related to the 
distribution of neutrons in the nucleus. The effect is tiny-only about one part per two million for the PREX experiment-so its measurement requires a heroic control of systematic uncertainties.

It is this minuscule parity-violating asymmetry that the PREX Collaboration has succeeded in measuring for lead-208, using the Jefferson Lab's high-resolution spectrometers. The experimenters scattered 953-MeV spin-polarized electrons off a lead foil sandwiched between thin layers of diamond. The polarization of the electrons was reversed hundreds of times per second, following a specific sequence whose details were hidden from the experimenters to reduce potential analysis bias. The measured excess in the right-handed electron-scattering cross section was $550 \pm 18$ parts per billion, with most of the uncertainty contributed by statistical error. From these data, the team inferred a "neutron radius" (the radius of the neutrons' distribution within the nucleus; not that of individual neutrons) of $5.800 \pm 0.075 \mathrm{fm}$. Given the proton radius value established by previous experiments, this measurement showed the neutron skin width to be $0.283 \pm 0.071 \mathrm{fm}-$ a twofold improvement in precision over the collaboration's earlier estimate [2].

As tiny as these dimensions are, their implications are astronomical: measuring the neutron skin in a single nucleus (0.2-fm scale) can inform our knowledge of neutron star structure ( $\mathrm{km}$ scale). The link between these objects works through a quantity called the symmetry energy-a contribution to the nuclear binding energy that arises because of the Pauli principle in nuclei that have unequal numbers of neutrons and protons-and a related phenomenon called symmetry pressure [3]. If the symmetry energy increases rapidly as the nuclear density increases, the symmetry pressure will be larger. In a nucleus, a larger symmetry pressure means neutrons are pushed out farther, yielding a thicker neutron skin. Similarly, in a neutron star, a higher symmetry pressure correlates with a larger radius for a given mass.

These neutron star properties hinted at by the PREX measurements will affect how we interpret observations of binary neutron star mergers, which are now routinely detected by gravitational-wave interferometers. Gravitational-wave signals can reveal how matter is deformed during such collisions [4], but the details depend on how large a neutron star is for a given mass. Insight from the PREX result therefore informs our understanding of these cataclysmic events. A new study already shows that theoretical expectations for the symmetry pressure are systematically a bit low compared with the value inferred from PREX, although the experimental result is still consistent with predictions within uncertainties [5]. This study also finds that PREX is in mild tension with gravitational-wave determinations of neutron star deformability. Future gravitational-wave and $\mathrm{x}$-ray observations will help clarify the picture $[3,6]$.

Precise measurements of the neutron skin thickness could also lead to new discoveries in particle physics. Neutrinos interact rarely with nuclei, but when they do, they can coherently scatter off an entire nucleus via the exchange of a $Z$ boson, giving the nucleus a gentle kick [7]. The distribution of nuclear recoil energies depends on the arrangement of the neutrons in the nucleus; any anomalies in the energy distribution can be used to test for new physics. Although the rarity of such neutrino interactions limits the effectiveness of present-generation experiments, for next-generation, high-statistics tests of beyond-the-standard-model neutrino interactions, precise understanding of neutron spatial distributions within the nucleus will reduce ambiguities [8].

There are several present and future prospects for nuclear-scattering-based neutron skin measurements that will complement the recent result from the PREX Collaboration. A similar polarized-electron-scattering measurement on calcium-48, called the Calcium Radius Experiment (CREX), was recently performed at the Jefferson Lab, and the data are in the process of being analyzed [9], while an improved measurement on lead-208 is planned at an accelerator facility in Mainz, Germany [10]. Strong-force-based measurements on rare nuclei are planned for the Facility for Rare Isotope Beams at Michigan State University [11]. And in a different approach, future observations using gravitational waves, $x$ rays, and neutrinos have exciting potential to shine diverse kinds of "light" on this story of nuclear structure connections over vastly different scales.

Kate Scholberg: Physics Department, Duke University, Durham, NC, USA 


\section{REFERENCES}

1. D. Adhikari et al. (PREX Collaboration), "Accurate determination of the neutron skin thickness of ${ }^{208} \mathrm{~Pb}$ through parity-violation in electron scattering," Phys. Rev. Lett. 126, 172502 (2021).

2. S. Abrahamyan et al. (PREX Collaboration), "Measurement of the neutron radius of ${ }^{208} \mathrm{~Pb}$ through parity violation in electron scattering," Phys. Rev. Lett. 108, 112502 (2012).

3. J. Piekarewicz and F. J. Fattoyev, "Neutron-rich matter in heaven and on Earth," Phys. Today 72, 30 (2019).

4. B. P. Abbott et al. (LIGO Scientific Collaboration and Virgo Collaboration), "GW170817: Observation of gravitational waves from a binary neutron star inspiral," Phys. Rev. Lett. 119, 161101 (2017).

5. B. T. Reed et al., "Implications of PREX-II on the equation of state of neutron-rich matter," Phys. Rev. Lett. 126, 172503 (2021).
6. G. Raaijmakers et al., "Constraining the dense matter equation of state with joint analysis of NICER and LIGO/Virgo measurements," Astrophys. J. Lett. 893, L21 (2020).

7. D. Akimov et al. (Coherent Collaboration), "Observation of coherent elastic neutrino-nucleus scattering," Science 357, 1123 (2017).

8. D. Aristizabal Sierra et al., "Impact of form factor uncertainties on interpretations of coherent elastic neutrino-nucleus scattering data," J. High Energy Phys. 6, 141 (2019).

9. J. Mammei et al. (CREX Collaboration), Proposal to Jefferson Lab PAC 40, CREX: Parity-violating measurement of the weak charge distribution of ${ }^{48} \mathrm{Ca}$ to $0.02 \mathrm{fm}$ accuracy (unpublished).

10. D. Becker et al., "The P2 experiment,” Eur. Phys. J. A 54, 208 (2018).

11. The 2015 Nuclear Advisory Committee, "Reaching for the horizon: The 2015 long range plan for nuclear science”. 